\title{
Detection of lithium in nearby young late-M dwarfs ${ }^{\star}$
}

\author{
N. Phan-Bao ${ }^{1,2}$, M. S. Bessell ${ }^{3}$, D. Nguyen-Thanh ${ }^{1,4}$, E. L. Martín ${ }^{5}$, P. T. P. Ho ${ }^{2}$, C. F. Lee ${ }^{2}$, and H. Parsons ${ }^{6}$ \\ ${ }^{1}$ Department of Physics, HCM International University-Vietnam National University, Block 6, Linh Trung Ward, Thu Duc District, \\ HCM city, Viet Nam \\ e-mail: pbngoc@hcmiu.edu.vn \\ 2 Institute of Astronomy and Astrophysics, Academia Sinica. PO Box 23-141, Taipei 106, Taiwan, ROC \\ 3 Research School of Astronomy and Astrophysics, Australian National University, Cotter Rd, 2611 Weston, Australia \\ ${ }^{4}$ Faculty of Physics and Engineering Physics, HCM University of Science-VNU, 227 Nguyen Van Cu Street, District 5, \\ HCM city, Viet Nam \\ 5 Centro de Astrobiologia (CSIC-INTA), Ctra. Ajalvir km 4, 28850 Torrejón de Ardoz, Madrid, Spain \\ ${ }^{6}$ East Asian Observatory, 660 N. A’Ohoku Place University Park, Hilo, Hawaii 96720, USA
}

Received 24 September 2016 / Accepted 29 December 2016

\begin{abstract}
Context. Late M-type dwarfs in the solar neighborhood include a mixture of very low-mass stars and brown dwarfs that is difficult to disentangle due to the lack of constraints on their age, such as trigonometric parallax, lithium detection, and space velocity.

Aims. We search for young brown dwarf candidates among a sample of 28 nearby late-M dwarfs with spectral types between M5.0 and M9.0, and we also search for debris disks around three of them.

Methods. Based on theoretical models, we used the color $I-J$, the $J$-band absolute magnitude, and the detection of the Li I $6708 \AA$ doublet line as a strong constraint to estimate masses and ages of our targets. For the search of debris disks, we observed three targets at submillimeter wavelength of $850 \mu \mathrm{m}$.

Results. We report here the first clear detections of lithium absorption in four targets and a marginal detection in one target. Our mass estimates indicate that two of them are young brown dwarfs, two are young brown dwarf candidates, and one is a young very low-mass star. The closest young field brown dwarf in our sample at only $\sim 15 \mathrm{pc}$ is an excellent benchmark for further studying physical properties of brown dwarfs in the range 100-150 Myr. We did not detect any debris disks around three late-M dwarfs, and we estimated upper limits to the dust mass of debris disks around them.
\end{abstract}

Key words. brown dwarfs - radio continuum: stars - techniques: spectroscopic - circumstellar matter - techniques: photometric stars: flare

\section{Introduction}

Since the discovery of the first lithium-bearing late-M substellarmass members in the benchmark Pleiades cluster (Rebolo et al. 1995, 1996; Basri et al. 1996; Martín et al. 1996), hundreds of brown dwarfs (BD, 13-75 $M_{\mathrm{J}}$ ) and very low-mass (VLM, 0.075$0.35 M_{\odot}$ ) stars have been identified in the field and in young open clusters and star-forming regions.

According to the theory of VLM objects, a BD with a mass below $\sim 60 M_{\mathrm{J}}$ should never reach a high enough core temperature to destroy its primordial lithium content (Magazzù et al. 1993). Stars with masses below the mass limit of $\sim 0.35 M_{\odot}$ (spectral types of $\sim \mathrm{M} 3-\mathrm{M} 4$ ) are predicted to be fully convective (Chabrier \& Baraffe 2000). Because BDs have masses well below this mass limit, if lithium is not destroyed in the BD core, it should be detected in their atmosphere as initially proposed in Rebolo et al. (1992). However, the lithium depletion depends not only on stellar mass but also on age, metallicity (Chabrier et al. 1996), and rotation (e.g., Messina et al. 2016). Some G, K, and early-M dwarfs at young ages also exhibit strong lithium absorption (Bopp 1974; Favata et al. 1997; Song et al. 2002; Murphy \& Lawson 2015). The combination of mass with

\footnotetext{
$\star$ The reduced spectra (FITS files) are only available at the CDS via anonymous ftp to cdsarc.u-strasbg. fr (130.79.128.5) or via http://cdsarc.u-strasbg.fr/viz-bin/qcat?J/A+A/600/A19
}

age and lithium depletion will set up a temperature boundary below which an object must be substellar if lithium is detected. Basri (2000) has shown that if lithium is present in any objects with effective temperatures below $2790 \mathrm{~K}$, which corresponds to a spectral type of $\sim$ M6 (e.g., Rajpurohit et al. 2013), these objects should be BDs (see Fig. 1 in the Basri paper). Therefore, late-M dwarfs with spectral types of M6 or later that show the Li I resonance doublet line at $6708 \AA$ in their spectrum should be BDs.

Lithium as an age indicator has been used to search for young BDs among nearby late-M dwarfs. Martín et al. (1994) looked for lithium in 12 field late-M dwarfs without success. The first three lithium detections in field late-M dwarfs were reported in Thackrah et al. (1997), Tinney (1998), Martín et al. (1999a). Reid et al. (2002) searched for lithium in 39 dwarfs with spectral type in the range M6.5-L0.5 and found strong lithium absorption in two late-M dwarfs. Reiners \& Basri (2009) searched for lithium in a sample of 63 late-M dwarfs and reported detections in six of them. Those searches for lithium among field late$\mathrm{M}$ dwarfs indicate that approximately $10 \%$ of them are young brown dwarfs.

In this paper, we present a search for lithium in a sample of 28 nearby late-M dwarfs (spectral types from M5.0 to M9.0) selected on the basis of relatively strong $\mathrm{H} \alpha$ emission in lowresolution spectra. The Li I resonance doublet line centered at 


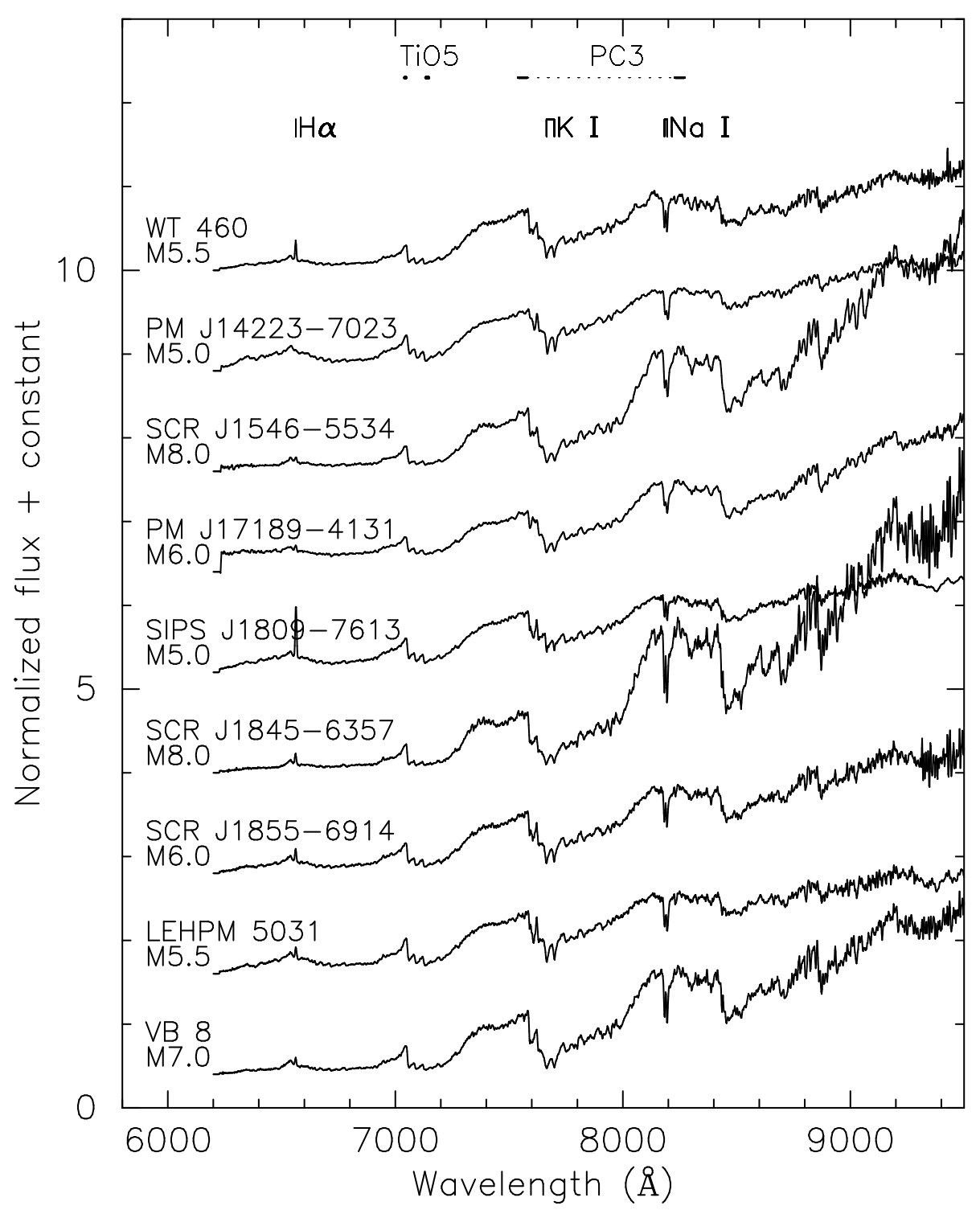

Fig. 1. Low-resolution spectra of 8 very nearby late-M dwarfs and the VB 8 reference as listed in Table 2. The H $\alpha$, Na I, K I lines and the spectral intervals used for calculating the TiO5 and PC3 indices are indicated.

$6708 \AA$ is clearly detected in four and marginally detected in one of our targets. We then used theoretical models to estimate the mass and the age of these lithium BD candidates. These nearby $\mathrm{BD}$ candidates are benchmarks for further studies of the basic properties of young substellar-mass objects.

The rest of this paper is organized as follows: We present our selection of targets in Sect. 2 and the spectroscopic observations in Sect. 3. In Sect. 4, we estimate spectral types and spectroscopic distances of ten late-M dwarfs within $13 \mathrm{pc}$ and then present the lithium detections and equivalent width measurement of $\mathrm{H} \alpha$ and the $\mathrm{Na}$ I doublet at $8183 \AA$ and $8199 \AA$ in our targets. We also discuss the variability of $\mathrm{H} \alpha$ emission and report a strong flare observed in an M7.5 dwarf. In Sect. 5, we estimate the masses and the ages of the five dwarfs with detected lithium and present our search for debris disks around three targets. Section 6 summarizes our results.

\section{Targets}

The presence of lithium in late-M dwarfs indicates that the sources are young and their masses are substellar or very close to the substellar boundary. We therefore selected late-M dwarfs with spectral types $\geq$ M5.0 (see Table 1). These late-M dwarfs were identified from the DENIS survey (see Phan-Bao et al. 2001, 2003; Phan-Bao \& Bessell 2006; Crifo et al. 2005) and have estimated spectral types as well as spectroscopic distances (Phan-Bao \& Bessell 2006; Crifo et al. 2005). Most of the selected targets show relatively strong $\mathrm{H} \alpha$ emission, an indicator of magnetic activity.

Young late-M dwarfs are magnetically active and thus show strong $\mathrm{H} \alpha$ emission. However, in late-M dwarfs, the magnetic activity depends not only on their age but also on the dynamo mechanism operating in these fully convective stars (e.g., see Phan-Bao et al. 2009, and references therein). In addition, West et al. (2008) have suggested that the lifetimes of magnetic activity of late-M dwarfs may be a few Gyr. Many old late-M dwarfs also show strong $\mathrm{H} \alpha$ emission. Therefore, one should note that the presence of $\mathrm{H} \alpha$ emission in our late-M dwarfs cannot confirm their youth but it implies that the selected targets are potential young late-M dwarf candidates.

In our sample, we also included very nearby late-M dwarfs that we had identified in the DENIS database (see 
Table 1. H $\alpha$, Li I $6708 \AA$ and Na I (8170-8200 ̊) equivalent widths of 28 nearby late-M dwarfs.

\begin{tabular}{|c|c|c|c|c|c|c|c|c|}
\hline DENIS-P name & Other name & SpT & $\begin{array}{l}\text { Distance } \\
(\mathrm{pc})\end{array}$ & $\begin{array}{l}\text { UT observing } \\
\text { date }\end{array}$ & $\begin{array}{l}E W \mathrm{H} \alpha \\
(\AA)\end{array}$ & $\begin{array}{l}E W \mathrm{Li} \\
(\AA)\end{array}$ & $\begin{array}{l}E W \mathrm{Na} I \\
(\AA)\end{array}$ & References \\
\hline $\mathrm{J} 0041353-562112^{a}$ & & M7.5 & $17.0 \pm 2.4$ & $2008-03-29$ & $-16.1 \pm 0.4$ & $<0.2$ & $3.3 \pm 0.6$ & (1) \\
\hline J0103119-535143 & & M5.5 & $24.3 \pm 3.7$ & 2008-03-29 & $-8.9 \pm 0.1$ & $<0.1$ & $5.0 \pm 0.1$ & (1) \\
\hline J0144318-460432 & & M5.5 & $23.3 \pm 3.4$ & $2005-07-29$ & $-12.1 \pm 0.2$ & $0.4 \pm 0.1$ & $3.5 \pm 0.6$ & (1) \\
\hline J0253444-795913 & & M5.5 & $17.2 \pm 2.4$ & $2005-07-29$ & $-6.5 \pm 0.1$ & $<0.2$ & $5.7 \pm 0.6$ & (1) \\
\hline J0334113-495333 & & M9.0 & $8.2 \pm 0.8$ & 2005-11-09 & $>-0.1$ & $<0.1$ & $6.7 \pm 0.1$ & (2) \\
\hline J0351000-005244 & LHS 1604 & M7.0 & $12.8 \pm 1.8$ & 2006-01-10 & $-5.9 \pm 0.4$ & $1.2 \pm 0.2$ & $7.6 \pm 0.5$ & (3) \\
\hline J0410480-125142 & LP $714-37^{b}$ & M5.5 & $18.1 \pm 2.2$ & $2008-03-28$ & $-0.3 \pm 0.1$ & $<0.1$ & $7.7 \pm 0.4$ & (4) \\
\hline J0440231-053009 & LP 655-48 & M7.5 & $8.9 \pm 1.3$ & $2008-03-28$ & $-35.2 \pm 0.2$ & $<0.1$ & $8.5 \pm 0.5$ & (3) \\
\hline J0517377-334903 & & M8.0 & $12.1 \pm 1.8$ & $2008-03-28$ & $-3.9 \pm 1.2$ & $<0.3$ & $5.9 \pm 0.6$ & (1) \\
\hline J0518113-310153 & & M6.5 & $19.5 \pm 2.9$ & $2008-03-28$ & $-8.4 \pm 0.3$ & $0.6 \pm 0.3^{e}$ & $5.8 \pm 0.1$ & (3) \\
\hline J0740193-172445 & LHS 234 & M6.5 & $9.1 \pm 1.3$ & 2008-03-29 & $>-0.1$ & $<0.1$ & & this paper \\
\hline J0838022-585558 & SCR J0838-5855 & M6.0 & $11.3 \pm 1.6$ & $2008-03-29$ & $-3.2 \pm 0.1$ & $<0.1$ & & this paper \\
\hline J1236153-310646 & LP 909-55 & M5.5 & $19.4 \pm 2.7$ & $2008-03-28$ & $-8.3 \pm 0.2$ & $<0.1$ & $6.2 \pm 0.5$ & (1) \\
\hline $\mathrm{J} 1357149-143852$ & & M7.5 & $24.7 \pm 3.6$ & $2008-03-28$ & $-5.9 \pm 0.9$ & $<0.1$ & $6.2 \pm 0.8$ & (1) \\
\hline J1411599-413221 & $\mathrm{WT} 460^{c}$ & M5.5 & $10.1 \pm 1.3$ & $2008-03-28$ & $-6.2 \pm 0.1$ & $<0.1$ & $7.6 \pm 0.3$ & this paper \\
\hline $\mathrm{J} 1538317-103850$ & & M5.0 & $31.7 \pm 7.0$ & $2008-03-28$ & $-13.1 \pm 0.1$ & $<0.1$ & $4.3 \pm 0.9$ & (3) \\
\hline $\mathrm{J} 1553571-231152$ & LP 860-46 & M5.0 & $21.5 \pm 2.9$ & $2008-03-28$ & $-5.5 \pm 0.1$ & $<0.2$ & $7.2 \pm 0.5$ & (3) \\
\hline J1610584-063132 & LP 684-33 & M5.5 & $17.7 \pm 2.5$ & $2008-03-28$ & $-3.5 \pm 0.1$ & $<0.1$ & $8.3 \pm 0.1$ & (3) \\
\hline J1809068-761324 & SIPS J1809-7613 & M5.0 & $10.4 \pm 1.4$ & $2008-03-28$ & $-4.7 \pm 0.1$ & $0.5 \pm 0.1$ & $4.4 \pm 0.2$ & this paper \\
\hline $\mathrm{J} 1845049-635747$ & SCR J1845-6357 ${ }^{d}$ & M8.0 & $3.2 \pm 0.4$ & $2008-03-28$ & $-2.1 \pm 0.1$ & $<0.1$ & $9.0 \pm 0.5$ & this paper \\
\hline J1855480-691415 & SCR J1855-6914 & M6.0 & $11.0 \pm 1.6$ & $2008-03-28$ & $-3.5 \pm 0.1$ & $<0.1$ & $7.6 \pm 0.3$ & this paper \\
\hline J1917045-301920 & LP 924-17 & M5.5 & $22.1 \pm 3.1$ & $2008-03-28$ & $-8.5 \pm 0.2$ & $<0.1$ & $8.3 \pm 0.2$ & (3) \\
\hline J2002134-542555 & & M6.0 & $17.5 \pm 2.5$ & $2005-07-29$ & $-3.4 \pm 0.1$ & $<0.1$ & $5.6 \pm 0.1$ & (1) \\
\hline J2022480-564556 & & M5.5 & $22.9 \pm 3.3$ & $2008-03-28$ & $-5.3 \pm 0.2$ & $0.4 \pm 0.1$ & $5.0 \pm 0.8$ & (3) \\
\hline J2049527-171608 & LP 816-10 & M6.0 & $19.4 \pm 5.7$ & $2008-03-28$ & $-5.3 \pm 0.2$ & $<0.1$ & $8.6 \pm 0.6$ & (3) \\
\hline J2132297-051158 & LP 698-2 & M5.5 & $18.5 \pm 2.6$ & $2005-07-29$ & $>-0.1$ & $<0.2$ & $5.5 \pm 0.2$ & (1) \\
\hline J2151270-012713 & LP $638-50$ & M5.0 & $18.7 \pm 3.1$ & $2005-07-29$ & $-2.8 \pm 0.1$ & $<0.1$ & $5.0 \pm 0.1$ & (1) \\
\hline J2241593-750034 & LEHPM 5031 & M5.5 & $12.5 \pm 1.8$ & $2008-03-28$ & $-1.9 \pm 0.1$ & $<0.1$ & $8.5 \pm 0.8$ & this paper \\
\hline
\end{tabular}

Notes. The $\mathrm{H} \alpha$ and Li I EWs measured from the medium-resolution spectra, and the NaI EWs measured from low-resolution spectra published in Crifo et al. (2005) and Phan-Bao \& Bessell (2006) (see Sect. 4.2). ${ }^{(a)}$ A young binary of M6.5+M9.0 (Reiners et al. 2010); ${ }^{(b)}$ a triple system of M5.5+M8.0+M8.5 (Phan-Bao et al. 2005, 2006b); ${ }^{(c)}$ a binary with photometric spectral types of M6.0+L1 estimated in Montagnier et al. (2006); ${ }^{(d)}$ a binary of M8.5+T5.5 (Henry et al. 2004; Biller et al. 2006); ${ }^{(e)}$ the lithium is marginally detected.

References. References for spectral type and distance: (1) Phan-Bao \& Bessell (2006); (2) Phan-Bao et al. (2006a); (3) Crifo et al. (2005); (4) Phan-Bao et al. (2005).

Phan-Bao et al. 2008 and references therein) with photometric distances within $12 \mathrm{pc}$ based on the $M_{\mathrm{I}}$ absolute magnitude versus $I-J$ color relationship in Phan-Bao et al. (2003). These dwarfs have been identified in high-proper-motion surveys (see Table 2), although several lack spectroscopic/trigonometric distances (SCR J0838-5855, PM J14223-7023, SCR J1546-5534, PM J17189-4131, SIPS J1809-7613, SCR J1855-6914 and LEHPM 5031) or spectroscopic spectral types (SCR J0838-5855, PM J17189-4131, SIPS J1809-7613, SCR J1855-6914 and LEHPM 5031).

\section{Spectroscopic observations}

We observed 28 late-M dwarfs from 2005 to 2008 with the double-beam grating spectrograph (DBS) on the $2.3 \mathrm{~m}$ telescope at Siding Spring Observatory. The red channel of the DBS covers the wavelength range of $6480-7485 \AA$. The $1200 \mathrm{~g} / \mathrm{mm}$ grating was used, providing a medium-resolution of approximately $1.0 \AA$, at $0.5 \AA$ /pixel. Table 1 lists the observing date of our targets.

For 10 very nearby late-M dwarfs as listed in Table 2 (except LHS 234 and SCR J0838-5855), we observed them with DBS with the $316 \mathrm{~g} / \mathrm{mm}$ grating, which covers the wavelength range of $6200-10050 \AA$ at a low-resolution of approximately $4 \AA$, at $2 \AA /$ pixel.

We used FIGARO (Shortridge et al. 2004) to reduce the data. The data was flat fielded. No dark subtraction was done due to the insignificant dark current. No bad pixels were removed. One bad column was removed by interpolation. The 2D long slit spectrum of a bright star was traced and the image was transformed to straighten the spectrum. This transformation was then applied to each program star. All observations were made with the atmospheric dispersion along the slit so there is no curvature caused by the atmospheric dispersion. Extremely metal-deficient red giants with temperatures of $5000-6000 \mathrm{~K}$ and with $[\mathrm{Fe} / \mathrm{H}]<-2$ that make good smooth spectrum templates were observed during the night at air masses encompassing those for the targets to remove the telluric lines. These were then divided into the program star's spectrum and the residuals were examined. The best divided result was accepted. Each night one or two white dwarfs, such as EG 131, VMa 2, L745-46A, and LTT4364 were additionally observed to test whether or not the telluric removal was well carried out as they have no bands or lines redward of $4100 \AA$ and they are smooth blackbodies. After flat fielding and telluric absorption line removal, the standard star spectra in the red are very smooth and show slowly varying changes of 
Table 2. Spectral indices, distances and spectral types estimated for ten very nearby late-M dwarfs and the VB 8 reference observed at MSSSO$2.3 \mathrm{~m}$.

\begin{tabular}{llrlllllrll}
\hline \hline Name & $I$ & \multicolumn{1}{c}{$J$} & $K$ & $\begin{array}{l}\text { UT observing } \\
\text { date }\end{array}$ & VOa & \multicolumn{1}{l}{ TiO5 } & PC3 & \multicolumn{1}{l}{$\begin{array}{l}\text { Distance } \\
(\mathrm{pc})\end{array}$} & SpT $^{\mathrm{c}}$ & Ref. \\
\hline LHS 234 & 12.37 & 10.21 & 9.31 & $2008-03-29$ & & $0.15 \pm 0.03^{\mathrm{a}}$ & & $9.1 \pm 1.3^{\mathrm{b}}$ & $\mathrm{M} 6.5$ & 1 \\
SCR J0838-5855 & 12.77 & 10.34 & 9.20 & $2008-03-29$ & & $0.20 \pm 0.02^{\mathrm{a}}$ & & $11.3 \pm 1.6^{\mathrm{b}}$ & M6.0 & 2 \\
WT 460 & 11.77 & 9.64 & 8.59 & $2007-08-04$ & $2.06 \pm 0.14$ & $0.20 \pm 0.02^{\mathrm{a}}$ & $1.22 \pm 0.09$ & $10.1 \pm 1.3$ & M5.5 & 3 \\
PM J14223-7023 & 11.97 & 10.15 & 9.43 & $2008-03-27$ & $2.02 \pm 0.07$ & $0.36 \pm 0.08$ & $1.38 \pm 0.05$ & $10.0 \pm 1.4$ & M5.0 & 4 \\
SCR J1546-5534 & 12.96 & 10.22 & 9.14 & $2008-03-27$ & $2.22 \pm 0.15$ & $0.29 \pm 0.03$ & $2.05 \pm 0.10$ & $5.4 \pm 0.7$ & M8.0 & 5 \\
PM J17189-4131 & 12.95 & 10.55 & 9.60 & $2008-03-27$ & $2.10 \pm 0.09$ & $0.52^{\mathrm{d}}$ & $1.52 \pm 0.05$ & $10.5 \pm 1.5$ & M6.0 & 4 \\
SIPS J1809-7613 & 11.69 & 9.87 & 8.96 & $2007-08-04$ & $2.02 \pm 0.12$ & $0.26 \pm 0.03^{\mathrm{a}}$ & $1.25 \pm 0.06$ & $10.4 \pm 1.4$ & M5.0 & 6 \\
SCR J1845-6357 & 12.51 & 9.52 & 8.48 & $2007-08-04$ & $2.21 \pm 0.20$ & $0.28 \pm 0.03^{\mathrm{a}}$ & $2.42 \pm 0.18$ & $3.2 \pm 0.4$ & M8.0 & 7 \\
SCR J1855-6914 & 12.67 & 10.46 & 9.50 & $2007-08-04$ & $2.10 \pm 0.16$ & $0.22 \pm 0.02^{\mathrm{a}}$ & $1.43 \pm 0.09$ & $11.0 \pm 1.6$ & M6.0 & 8 \\
LEHPM 5031 & 12.54 & 10.39 & 9.54 & $2007-08-04$ & $2.04 \pm 0.11$ & $0.24 \pm 0.03^{\mathrm{a}}$ & $1.32 \pm 0.11$ & $12.5 \pm 1.8$ & M5.5 & 9 \\
VB 8 & 12.24 & 9.74 & 8.82 & $2007-08-04$ & $2.15 \pm 0.18$ & $0.18 \pm 0.01$ & $1.74 \pm 0.11$ & $5.8 \pm 0.8$ & M7.0 & \\
\hline
\end{tabular}

Notes. The VOa, TiO5 and PC3 indices measured from the low-resolution spectra, except TiO5 for some cases as noted (see Sect. 4.1). ${ }^{(a)}$ The TiO5 index measured from medium-resolution spectra. ${ }^{(b)}$ Distances estimated from the spectral type versus magnitude relation in Filippazzo et al. (2015). ${ }^{(c)}$ An uncertainty of 0.5 subtypes of estimated spectral types. ${ }^{(d)}$ An unreliable value, not being used to estimate spectral type.

References. References for the source name: (1) Luyten (1979); (2) Finch et al. (2007); (3) Wroblewski \& Torres (1991); (4) Lépine (2008); (5) Boyd et al. (2011); (6) Deacon \& Hambly (2007); (7) Hambly et al. (2004); (8) Subasavage et al. (2005); (9) Pokorny et al. (2003).

continuum intensity with wavelength. These standards were also used for flux calibration and a NeAr arc for wavelength calibration. The details of the technique were given in Bessell (1999).

The signal-to-noise ratios are in the range of 7-23 for medium-resolution spectra, except four spectra (J0041353562112, J0103119-535143, J0517377-334903, and J1357149143852 ) with moderate values of 3-5. For low-resolution spectra, the signal-to-noise ranges from 8 to 20 .

\section{Results}

\subsection{Estimate of spectral types and distances of ten very nearby late- $M$ dwarfs}

Based on both low- and medium-resolution spectra, we estimated the spectral types of the ten very nearby late-M dwarfs (Table 2). Spectral types of M dwarfs could be determined using spectral indices PC3 (Martín et al. 1999), TiO5, and VOa (see Cruz \& Reid 2002, and references therein), as described in detail in Phan-Bao \& Bessell (2006). The adopted spectral type is an average value of three spectral types estimated from the three spectral indices, except some cases as mentioned below. The PC3 and VOa indices were measured using low-resolution spectra. The TiO5 index is sensitive to the spectral resolution because the wavelength interval for the TiO5 denominator is very narrow (only $4 \AA$, 7042-7046 $\AA$ ) and positioned at the head of a molecular band as explained in Crifo et al. (2005). We therefore used our medium-resolution spectra available for seven targets (Table 2) to measure TiO5. For the three remaining targets, low-resolution spectra were used to determine TiO5. We did not observe LHS 234 or SCR J0838-5855 at low-resolution spectroscopy, therefore, only the TiO5 index was used to estimate their spectral type. The PC3 and VOa indices are not available for LHS 234 and SCR J0838-5855 as the observed wavelength of medium-resolution spectroscopy does not cover these indices.

To compute the distances, we used the PC3 index versus absolute magnitude relations in $I, J$, and $K$ bands (Crifo et al. 2005), or the spectral type versus $J$-band absolute magnitude relation (Filippazzo et al. 2015) for LHS 234 and SCR J0838-5855. Table 2 lists spectral types and distances and their associated errors estimated for these ten late-M dwarfs. One should note that these relations are applicable to field age late-M dwarfs. If our objects are young ( 10-150 Myr), our absolute magnitudes may be overestimated by $0.5-2.0$ mags (Faherty et al. 2012; Filippazzo et al. 2015), which results in the distances being underestimated by $23-92 \%$.

For the case of SCR J1546-5534 (or DENIS-P J1546418-553446, M8.0), our spectroscopic distance is only $5.4 \pm 0.7 \mathrm{pc}$, which is closer than the photometric distance of 6.7 pc estimated by Boyd et al. (2011).

\subsection{Lithium detection in five late-M dwarfs}

Based on medium-resolution spectra of 28 late-M dwarfs, we clearly detected the $\mathrm{Li}$ I resonance doublet line at $6708 \AA$ in four objects: DENIS-P J0144318-460432 (hereafter DENIS0144-4604), LHS 1604 (or DENIS-P J0351000005244), SIPS J1809-7613 (or DENIS-P J1809068-761324), and DENIS-P J2022480-564556 (hereafter DENIS20225645), as shown in Fig. 2. For the case of DENIS-P J0518113-310153 (hereafter DENIS0518-3101), the lithium is marginally detected. Using the IRAF task splot, we manually measured equivalent widths (EW) of the Li I line at $6708 \AA$. The continuum levels and integration limits were examined individually for each spectrum. The uncertainties in the EW measurement were derived by measuring EWs with different possible continuum levels as well as examining the noise around the region of 6700-6720 $\AA$. For the cases of non-detection of lithium, we measured upper limits by examining the noise in the region of interest. Our measurements are listed in Table 1.

Using the same approach as applied to the Li I EW measurement, we measured $\mathrm{H} \alpha$ EWs and their associated uncertainties for all targets. Four of the five late-M dwarfs with detected lithium showed a significant variation in $\mathrm{H} \alpha$ emission based on spectra obtained in this paper (see Tables 1 and 2), published in 


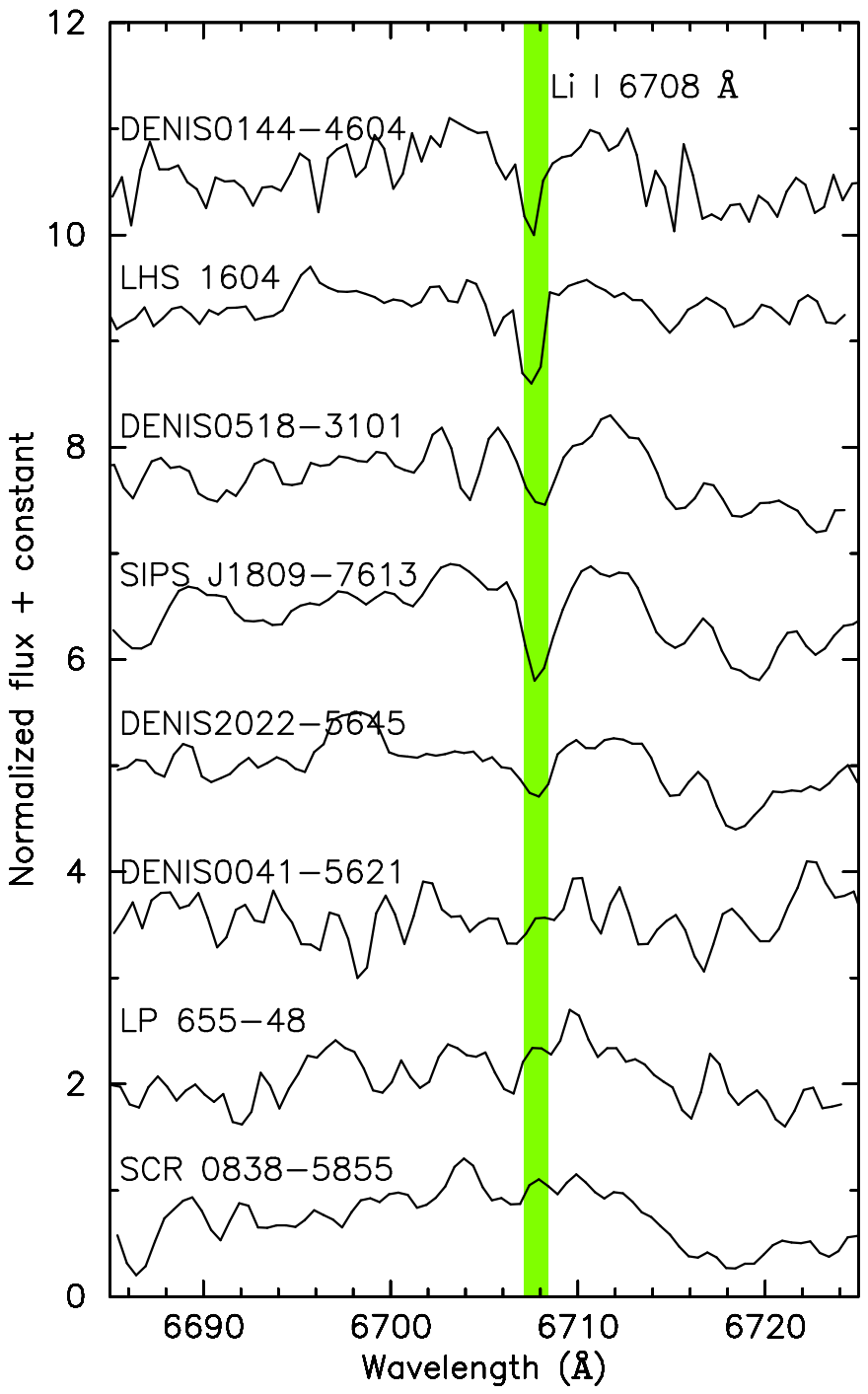

Fig. 2. Medium-resolution spectra of five late-M dwarfs with detected lithium (five upper spectra). The spectra of three late-M dwarfs with non-detection of lithium are also shown (three lower spectra). The region of the Li I resonance doublet line at $6708 \AA$ (Pavlenko et al. 1995) is indicated.

Crifo et al. (2005), Phan-Bao \& Bessell (2006) and reported in the literature at different epochs (UT time) as follows:

- DENIS0144-4604 has $\mathrm{H} \alpha$ emission with $E W=$ $-12.1 \AA$ measured on 2005-07-29 and $-25.4 \AA$ on 2005-07-30 (Phan-Bao \& Bessell 2006).

- LHS 1604 has EW H $\alpha=-5.9 \AA$ measured on both 200601-10 and 2003-11-29 (Crifo et al. 2005) but $-11.8 \AA$ on 2003-10-18 (West et al. 2011).

- DENIS0518-3101 has EW H $\alpha=-8.4 \AA$ measured on 2008-03-28 and $-18.8 \AA$ on 2003-11-29 (Crifo et al. 2005).

- SIPS J1809-7613 has EW H $\alpha=-4.7 \AA$ measured on 200803-28 and $-23.2 \AA$ on 2007-08-04.

The variable $\mathrm{H} \alpha$ emission in these late-M dwarfs is possibly due to either flaring activity or their rotation (e.g., Berger et al. 2008a,b; Phan-Bao et al. 2009). For the case of DENIS2022-5645, its $\mathrm{H} \alpha$ emission was likely stable, with $E W=-5.3 \AA$ measured on 2008-03-28 and $-5.7 \AA$ on $2002-$ 08-06 (Crifo et al. 2005). The stable $\mathrm{H} \alpha$ emission suggests that this dwarf is (nearly) pole-on and there was no flare during the

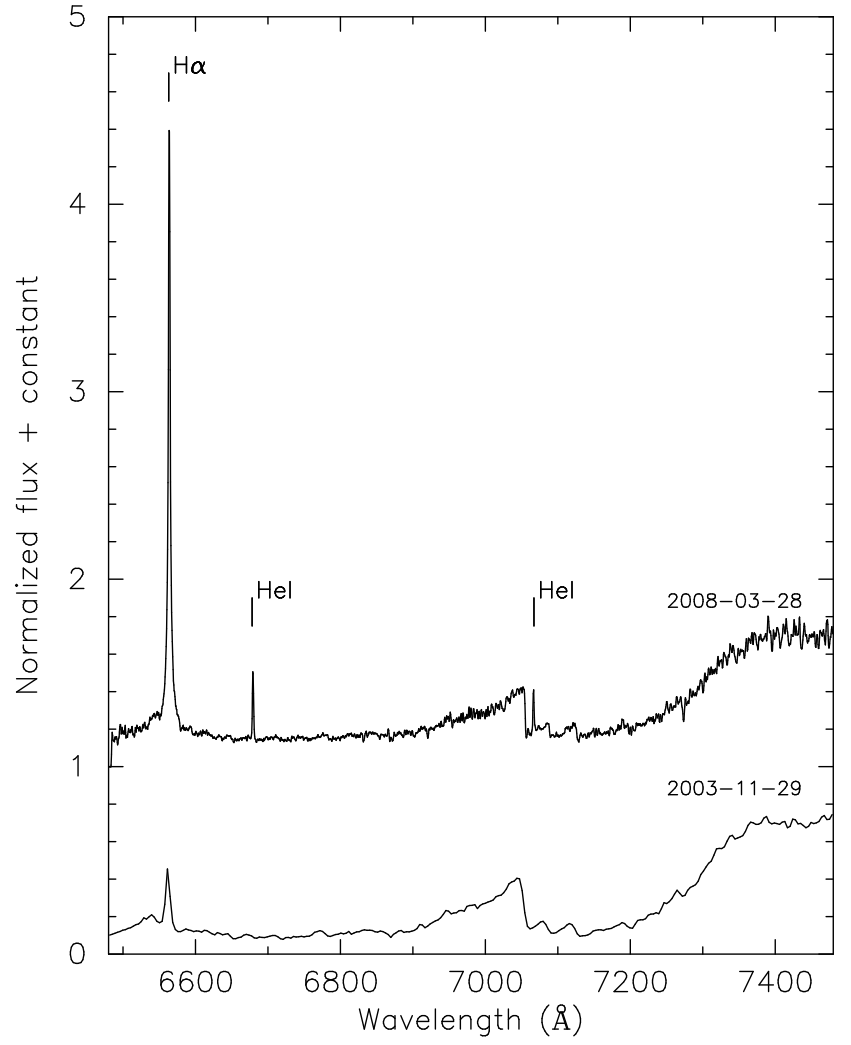

Fig. 3. LP 655-48 (M7.5, 9.5 pc) in a strongly flaring level with very strong $\mathrm{H} \alpha$ and $\mathrm{He}$ I emission lines (upper spectrum, this paper) and in a lower level of activity (lower spectrum, Crifo et al. 2005). The observing dates are also indicated.

observations. Spectroscopic monitoring of these five dwarfs for a full rotational period will clarify the possibilities.

In addition, we note a strong flare observed in LP 655-48 (M7.5) during our observations (see Fig. 3) with EW $\mathrm{H} \alpha=$ $-35.2 \AA$ measured on 2008-03-28. This source has EW H $\alpha=$ -13.6 $\AA$ measured on 2003-11-29 from the spectrum taken in Crifo et al. (2005). This source also showed strong He I emission lines as seen in LP 412-31 (M8, Schmidt et al. 2007). One should mention here that we detected no lithium in the source (Fig. 2), which is in agreement with the non-detection of lithium as reported in Reiners \& Basri (2009). LP 655-48 has a trigonometric distance of $9.5 \pm 0.3 \mathrm{pc}$ (Shkolnik et al. 2012), which is in agreement with its spectroscopic distance of $8.9 \pm 1.3 \mathrm{pc}$ (Crifo et al. 2005). With $J=10.74$, using the trigonometric distance of the source, we then derived $M_{\mathrm{J}}=10.85$. According to the CIFIST2011 BT-Settl atmosphere models (Allard et al. 2013), the $J$-band absolute magnitude and the DENIS color $I-J=2.61$ of the source imply an age of $>400 \mathrm{Myr}$. This age estimate is likely consistent with the identification of LP $655-48$ as a candidate member of Hyades ( $600-800 \mathrm{Myr}$, Brandt \& Huang 2015) by Galvez-Ortiz et al. (2010). At an age $>400$ Myr, the source should have no lithium (see also Fig. 5). This is consistent with the non-detections of lithium but disagrees with the detection of weak lithium absorption as reported in Shkolnik et al. (2009) and an age range of 10-90 Myr estimated in Shkolnik et al. (2012). One should note that the source has a radial velocity of $31.1 \pm 1.4 \mathrm{~km} \mathrm{~s}^{-1}$ (Deshpande et al. 2012). Using BANYAN $\mathrm{II}^{1}$, which is a Bayesian analysis tool for

1 http://www . astro.umontreal.ca/ gagne/banyanII.php 


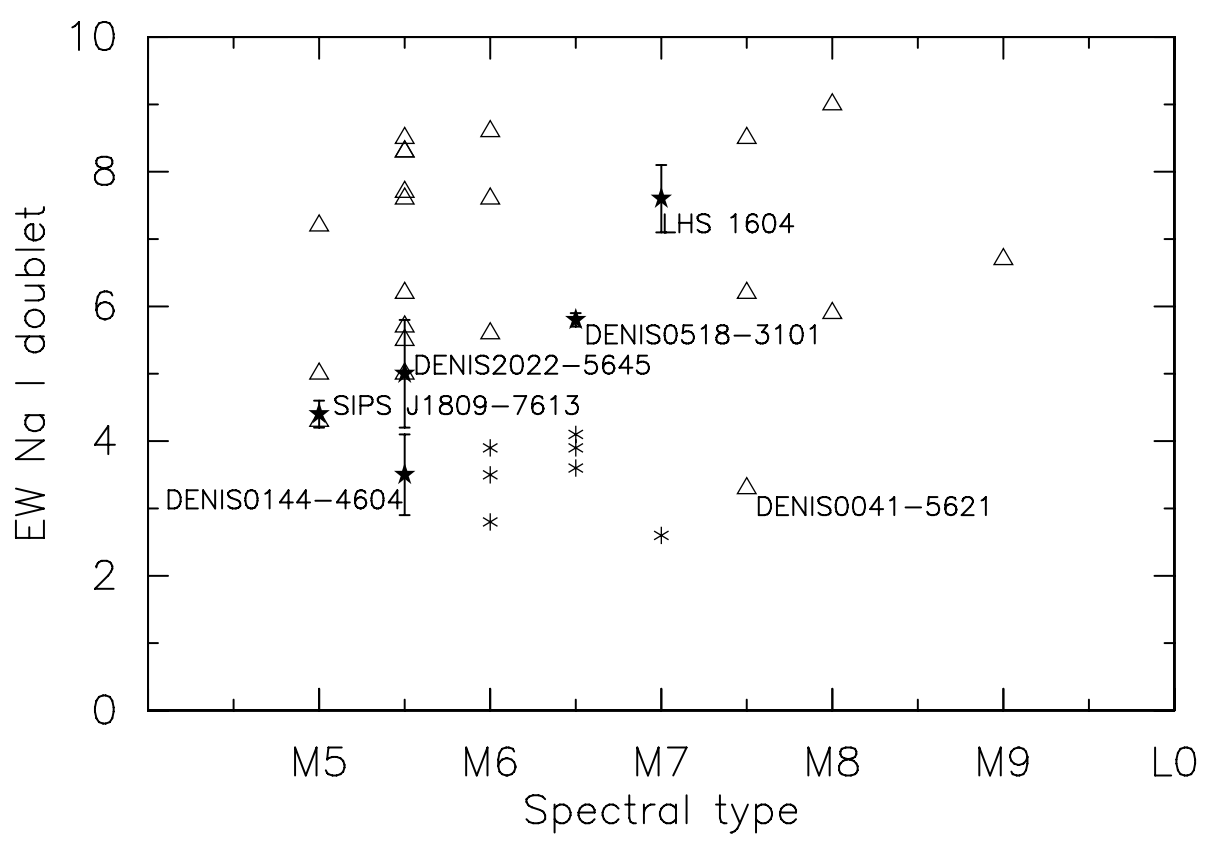

Fig. 4. Na I (8183/8199 ^) equivalent width versus spectral type diagram for our 28 late-M dwarfs and 7 Upper Sco candidate members (asterisk symbols, Martín et al. 2010). Five late-M dwarfs (star symbols) with detected lithium in this paper are shown as well as DENIS0041-5621 with lithium detection reported in Reiners \& Basri (2009) and Burgasser et al. (2015). estimating the membership probability of candidates to nearby young moving groups (see Gagné et al. 2014; Malo et al. 2013), and the proper motion of LP 655-48 in Phan-Bao et al. (2003), we found that the object has a $75.11 \%$ probability of being a member of old field (age $>1$ Gyr). Therefore, LP 655-48 is very likely an old field late-M dwarf.

Using the spectra of 28 late-M dwarfs published in Crifo et al. (2005) and Phan-Bao \& Bessell (2006), we also measured EWs of the Na I doublet at $8183 \AA$ and $8199 \AA$, which is an indicator for surface gravity of young $M$ dwarfs (e.g., Lyo et al. 2004; Schlieder et al. 2012), over the range of 8170-8200 $\AA$ as described in Martín et al. (2010). The uncertainties in the EW measurement were estimated by measuring EWs with different possible continuum levels. Our measurements are listed in Table 1. Figure 3 shows the Na I EW versus spectral type relation for our late-M dwarfs. Three of five dwarfs with detected lithium show weak $\mathrm{Na}$ I: DENIS0144-4604, SIPS J1809-7613 and DENIS2022-5645. Their Na I EWs are comparable to those of seven Upper Sco candidates measured in Martín et al. (2010). This implies that they have low surface gravity. The $\mathrm{Na}$ I EW of DENIS0144-4604 ( $E W=3.5 \AA$, M5.5) is comparable to that of DENIS0041-5621 ( $E W=3.3 \AA$, M7.5). At this point, one should note that DENIS0041-5621, which was identified as an M7.5 (Phan-Bao et al. 2001; Phan-Bao \& Bessell 2006), is actually a binary of M6.5+M9.0 (Reiners et al. 2010) with lithium detection as reported in Reiners \& Basri (2009) and Burgasser et al. (2015) $(E W=0.7 \AA)$. However, we did not detect lithium in DENIS0041-5621 (see Fig. 3) with an upper limit of $0.2 \AA$. The non-detection of lithium in the source is probably due to its low spectral signal-to-noise ratio of only $\sim 3$. We therefore cannot confirm the previous detections of lithium in DENIS0041-5621 with our current data. The two remaining targets, LHS 1604 and DENIS0518-3101, show Na I EWs significantly higher than those of the three objects above. For the case of LHS 1604, no lithium has been detected in the previous observations (Reiners \& Basri 2009; Burgasser et al. 2015). However, we detected a strong lithium absorption line with $E W=1.2 \pm 0.2 \AA$ (see Fig. 2) which is right at the upper limit of $1.2 \AA$ as reported in Burgasser et al. (2015).

\section{Discussion}

The detection of the $\mathrm{Li}$ I $6708 \AA$ doublet line in five late-M dwarfs DENIS0144-4604 (M5.5), LHS 1604 (M7.0), DENIS0518-3101 (M6.5), SIPS1809-7613 (M5.0), and DENIS2022-5645 (M5.5) indicates that these dwarfs are possibly young BDs. In order to determine the substellar nature of these objects, we used the CIFIST2011 BT-Settl atmosphere models (Allard et al. 2013) for the DENIS photometric system to estimate their mass and age range.

\subsection{Estimate of mass and age range of five young VLM objects}

DENIS0144-4604 (M5.5): this late-M dwarf was discovered by Phan-Bao et al. (2003) and spectroscopically classified as an M5.5 in Phan-Bao \& Bessell (2006). No trigonometric parallax or radial velocity measurements have been reported. The detection of lithium in the object will place the source in the lithium region (see Fig. 5). This thus indicates that its real absolute magnitude should be brighter than the magnitude derived from its spectroscopic distance (see Table 1). According to the BT-Settl models, the lithium presence in DENIS0144-4604 and its color $I-J=2.19$ (Phan-Bao et al. 2003) indicate its age $\leq 120 \mathrm{Myr}$. This age constraint places an upper limit of $\sim 73 M_{\mathrm{J}}$ on the mass of DENIS0144-4604. The source is therefore a young BD. Using the BANYAN II tool and the proper motion measured in Phan-Bao et al. (2003), we found that DENIS0144-4604 has a $97.07 \%$ probability of being a member of the TucanaHorologium moving group and a kinematic distance of $40 \pm 3 \mathrm{pc}$. Assuming the source at this distance, with an apparent $J$-band magnitude of 11.91 (Phan-Bao et al. 2003) we then derived its absolute magnitude $M_{\mathrm{J}}=8.90$. Based on the BT-Settl models, with such an absolute magnitude, the object should have an age range of 10-20 Myr (Fig. 5). This age range however is not consistent with the Tucana-Horologium age of $45 \pm 4 \mathrm{Myr}$ (Bell et al. 2015). Therefore, measurements of radial velocity and/or parallax of DENIS0144-4604 are clearly needed to determine its mass precisely and its membership in nearby young moving groups. 


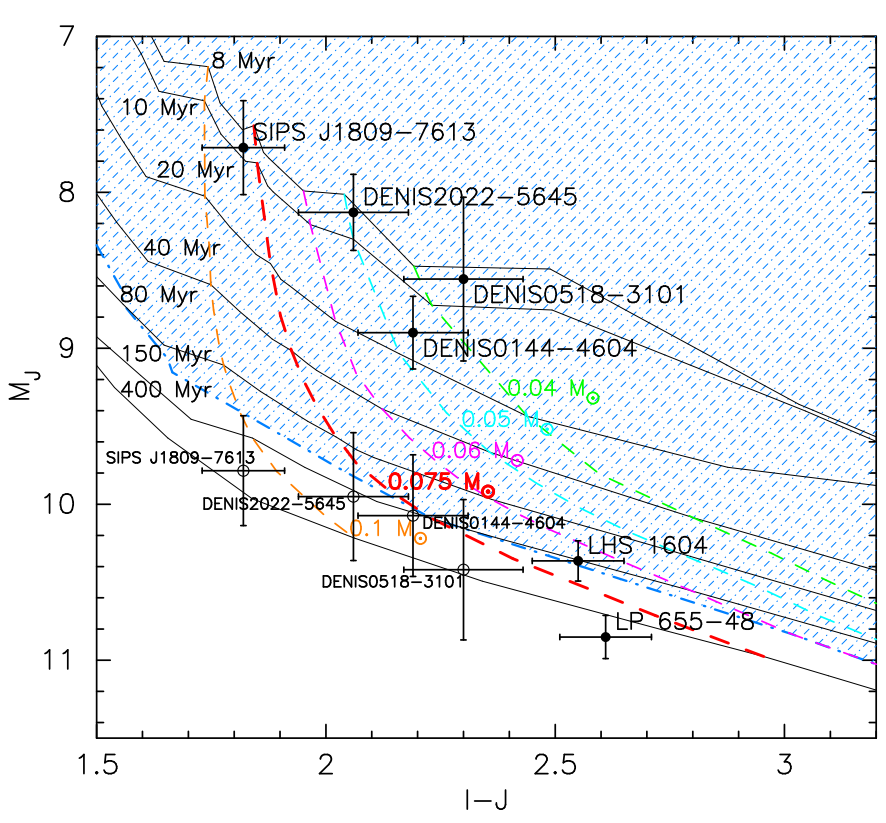

Fig. 5. $J$-band absolute magnitude versus color $I-J$ diagram for the five late-M dwarfs with detected lithium. Isochrones and mass tracks from the CIFIST2011 BT-Settl atmosphere models (Allard et al. 2013) are plotted. The lithium region is marked with the blue hatched area For the objects with no parallax measurements (DENIS0144-4604, DENIS0518-3101, SIPS J1809-7613 and DENIS2022-5645), the open and solid circles represent their absolute magnitudes derived from spectroscopic and kinematic distances, respectively (see Sect. 5.1 for further details). LP 655-48 is also shown (see Sect. 4.2 for discussion).

LHS 1604 (M7.0): the source has a spectral type of M7.0 (e.g., Crifo et al. 2005) and a trigonometric parallax of $68.2 \pm$ 1.8 mas (Gliese \& Jahreiß 1991), which corresponds to a distance of $14.7 \pm 0.4 \mathrm{pc}$. The trigonometric distance of LHS 1604 is consistent with its spectroscopic distance of $12.8 \pm 1.8 \mathrm{pc}$ (Crifo et al. 2005). With $J=11.2$ (Phan-Bao et al. 2003) and the trigonometric distance, we derived $M_{\mathrm{J}}=10.36$. Based on the BT-Settl models, the detection of lithium in LHS 1604, its color $I-J=2.55$ (Phan-Bao et al. 2003), and its $J$-band absolute magnitude imply that the source has an age range of 100-150 Myr and a substellar mass (see Fig. 5). Using this age range and the $J$-band absolute magnitude of the source, we derived its mass to be 55-66 $M_{\mathrm{J}}$. We then adopt an average mass of $\sim 61 M_{\mathrm{J}}$ for the source. Using the BANYAN II tool, the proper motion measurement in Phan-Bao et al. (2003), and a radial velocity of $-11.9 \pm 2.0 \mathrm{~km} \mathrm{~s}^{-1}$ (Deshpande et al. 2012), we found that LHS 1604 has a $100 \%$ probability of being a member of young field. LHS 1604 is therefore a young field BD within $15 \mathrm{pc}$.

DENIS0518-3101 (M6.5): this low-proper motion dwarf was identified by Phan-Bao et al. (2003). It has a spectral type of M6.5 (Crifo et al. 2005). McLean et al. (2012) detected a strong radio emission at $8.5 \mathrm{GHz}$ from DENIS0518-3101. No trigonometric parallax and radial velocity of the source are available. Using the BT-Settl models, its color $I-J=2.3$ and our marginal detection of lithium suggest its age to be $\leq 150$ Myr. This age limit places an upper limit of $\sim 73 M_{\mathrm{J}}$ on the mass of the object. DENIS0518-3101 is therefore a young BD candidate. At this point, deeper observations are required to confirm the lithium presence in the object and thus to confirm its substellar nature. Using the BAYAN II tool and the low-proper motion measurement in Phan-Bao et al. (2003), we found that the source has a $93.3 \%$ membership probability of the Columba association and a kinematic distance of $46 \pm 9 \mathrm{pc}$. If the source is at this distance, its absolute magnitude will be $M_{\mathrm{J}}=8.55(\mathrm{~J}=11.87)$. However, according to the BT-Settl models, this absolute magnitude implies that the source should have an age range of 10-20 Myr (see Fig. 5) that is not consistent with an age of $42_{-4}^{+6}$ Myr determined for Columba (Bell et al. 2015). Therefore, measurements of radial velocity and/or parallax of DENIS0518-3101 are clearly needed to determine its membership in young moving groups.

SIPS J1809-7613 (M5.0): the source has an estimated spectral type of M5.0 and a spectroscopic distance of 10.4 pc (Table 2). No measurements of trigonometric parallax or radial velocity of the object have been reported so far. The clear detection of lithium in SIPS J1809-7613 will place the source in the lithium region (see Fig. 5). This therefore indicates that the real absolute magnitude of the source must be brighter than its magnitude derived from the spectroscopic distance. According to the BT-Settl models, the lithium presence in the object and its color $I-J=1.82$ (Table 2) indicate its age to be $\leq 80 \mathrm{Myr}$. This thus places an upper limit of $95 M_{\mathrm{J}}$ on the mass of SIPS J1809-7613. Using the proper motion measurement from Deacon \& Hambly (2007), the BAYAN II tool implies that the object has a $76.2 \%$ membership probability of the $\beta$ Pic moving group and a kinematic distance of $27 \pm 3 \mathrm{pc}$. Assuming the source at this distance, with $J=9.87$ (Table 2), we then derived $M_{\mathrm{J}}=7.71$. With such a magnitude, the color-magnitude diagram (Fig. 5) suggests that the age of the object should be in the range of 10-20 Myr. This is likely consistent with the age of $\beta$ Pic of $24 \pm 3 \mathrm{Myr}$ (Bell et al. 2015). Using the estimated age range and the DENIS color $I-J$ of the source, we derived its mass to be $81-85 M_{\mathrm{J}}$. We then adopt an average mass of $83 M_{\mathrm{J}}$ for SIPS J1809-7613. The object is therefore a young VLM star.

DENIS2022-5645 (M5.5): this low-proper motion dwarf was discovered by Phan-Bao et al. (2003) and spectroscopically classified as an M5.5 in Crifo et al. (2005). The source has no trigonometric parallax and radial velocity measured so far. The presence of lithium in DENIS2022-5645 indicates that the object should locate in the lithium region as shown in Fig. 5. Therefore, the real absolute magnitude of the source must actually be brighter than its magnitude derived from the spectroscopic distance. Based on the BT-Settl models, our Li I detection in the source and its DENIS color $I-J=2.06$ (Phan-Bao et al. 2003) indicate that the source age should be $\leq 120$ Myr. This age constraint places an upper limit of $\sim 79 M_{\mathrm{J}}$ on the mass of DENIS2022-5645, which is very close to the substellar boundary. The source is therefore a young BD candidate. Using the proper motion measurement in Phan-Bao et al. (2003) and the BAYAN II tool, we found that the object has a 59.9\% probability of being a member of Tucana-Horologium and a kinematic distance of $53 \pm 4 \mathrm{pc}$. Assuming the source at this kinematic distance, we then derived $M_{\mathrm{J}}=8.13(J=11.75)$. With such an absolute magnitude, the BT-Settl models (Fig. 5) indicate that the source should have an age of $\leq \sim 10 \mathrm{Myr}$. This age estimate, however, disagrees with the age of $45 \pm 4$ Myr determined for Tucana-Horologium (Bell et al. 2015). Therefore, radial velocity and/or parallax measurements are additionally required for DENIS2022-5645 to determine its membership in young moving groups and thus to confirm its substellar nature.

\subsection{A search for debris disks around three nearby VLM objects}

We also searched for debris disks around three late-M ( $\geq \mathrm{M} 7.0)$ and very nearby sources (LHS 1604, M7.0), LP 655-48 (M7.5), and DENIS0517-3349 (M8.0). LHS 1604 is a young field BD 
as discussed in Sect. 5.1. Debris disks are made of planetesimals left over from the process of planet formation. In debris disks, dust is continuously produced by collision and evaporation of planetesimals. Therefore, the detection of dust emission (i.e., debris disks) around relatively young dwarfs (ages $\geq \sim 10 \mathrm{Myr}$ ) implies the presence of larger bodies around the dwarfs (Wyatt 2008).

We observed the three targets at $850 \mu \mathrm{m}$ with the SCUBA-2 bolometer array (Holland al. 2013) at the James Clerk Maxwell Telescope. The data were obtained on 2016-03-31, 2016-04-01, and 2016-07-29 (UT time) during which time zenith opacities at $225 \mathrm{GHz}$ were in the range of $0.5-0.7$. The primary full width at half maximum (FWHM) beam is approximately $13^{\prime \prime}$ at the observed wavelength (Dempsey et al. 2013). The data were reduced using the Dynamic Iterative Map Maker (DIMM) in the SMURF package from the STAR-LINK software (Jenness et al. 2011) using the blank-field recipe designed specifically for faint emission (Chapin et al. 2013). Both CRL618 and Uranus were observed as flux calibrators and were found to be within the nominal values quoted by Dempsey et al. (2013). We note that the uncertainty in the absolute flux calibration is $\sim 5 \%$. We applied an additional correction factor of $10 \%$ to the data to compensate for the flux lost during match-filtering (as applied to the data when running the blank-field reduction, e.g., Chen et al. 2013).

We did not detect any dust emission at $850 \mu \mathrm{m}$ from the sources. We measured the rms of $2.7 \mathrm{mJy}, 5.0 \mathrm{mJy}$ and $4.4 \mathrm{mJy}$ for the continuum maps of LHS 1604, LP 655-48 and DENIS0517-3349, respectively. Based on 3 rms flux densities, we then used the formula for optically thin dust to estimate the upper limits to the dust mass of debris disks for $\mathrm{M}$ dwarfs (see Lestrade et al. 2006): $S_{\lambda}=M_{\mathrm{d}} \times B\left(\lambda, T_{\mathrm{g}}\right) \times \kappa_{850 \mu \mathrm{m}} / d^{2}$, where $T_{\mathrm{g}}$ is the dust temperature, $d$ is the distance to the source, and $\kappa_{850 \mu \mathrm{m}}=1.7 \mathrm{~cm}^{2} \mathrm{~g}^{-1}$ is the mass absorption coefficient of the dust at $850 \mu \mathrm{m}$ (Dent et al. 2000; Shirley et al. 2011). The trigonometric distances were used for LHS 1604 (14.7 pc, Gliese \& Jahreiß 1991) and LP 655-48 (9.5 pc, Shkolnik et al. 2012), and the spectroscopic distance for DENIS0517-3349 (12.1 pc, see Table 1). Assuming a mean dust temperature of $15 \mathrm{~K}$ (Lestrade et al. 2006), we then derived upper limits to the dust mass to be $\sim 4.3,3.3$, and 4.8 lunar masses for LHS 1604 , LP 655-48, and DENIS0517-3349, respectively.

\section{Conclusion}

In this paper, we report our detections of lithium in five late-M dwarfs. The results indicate a lithium detection rate of approximately $14 \%$. Using the theoretical models, we estimated their masses. Our mass estimates indicate that DENIS0144-4604 (M5.5) and LHS 1604 (M7.0) are young BDs, DENIS0518-3101 (M6.5) and DENIS2022-5645 (M5.5) are young BD candidates, and SIPS J1809-7613 (M5.0) is a young VLM star. LHS 1604 is a young field BD at only $15 \mathrm{pc}$ with an age range of 100-150 Myr. Measurements of radial velocity and trigonometric parallax are needed to confirm the substellar nature of the four remaining sources as well as their membership in young moving groups.

Acknowledgements. This research is funded by Vietnam National University HoChiMinh City (VNU-HCM) under grant number C2014-28-01. The research based on the JCMT data is funded by Vietnam National Foundation for Science and Technology Development (NAFOSTED) under grant number 103.99 2015.108. E.M. acknowledges funding from the Spanish Ministry of Economy and Competitiveness (MINECO) under grant AYA-2015-69350-C3-1. We would like to thank the referee for useful comments. The James Clerk Maxwell Telescope is operated by the East Asian Observatory on behalf of The National Astronomical Observatory of Japan, Academia Sinica Institute of Astronomy and Astrophysics, the Korea Astronomy and Space Science Institute, the National Astronomical Observatories of China and the Chinese Academy of Sciences (Grant No. XDB09000000), with additional funding support from the Science and Technology Facilities Council of the United Kingdom and participating universities in the United Kingdom and Canada. This research has made use of the VizieR catalogue access tool, CDS, Strasbourg, France. The original description of the VizieR service was published in A\&AS 143, 23. This research has made use of the SIMBAD database, operated at CDS, Strasbourg, France.

\section{References}

Allard, F., Homeier, D., Freytag, B., Schaffenberger, W., \& Rajpurohit, A. S. 2013, Mem. Soc. Astron. It., 24, 128

Basri, G. 2000, ARA\&A, 38, 485

Basri, G., Marcy, G. W., \& Graham, J. R. 1996, ApJ, 458, 600

Bell, C. P. M., Mamajek, E. E., \& Naylor, T. 2015, MNRAS, 454, 593

Bessell, M. S. 1999, PASP, 111, 1426

Berger, E., Gizis, J. E., Giampapa, M. S., Rutledge, R. E., et al. 2008a, ApJ, 673, 1080

Berger, E., Basri, G., Gizis, J. E., Giampapa, M. S., et al. 2008b, ApJ, 676, 1307 Biller, B. A., Kasper, M., Close, L. M., Brandner, W., \& Kellner, S. 2006, ApJ, 641, L141

Bopp, B. W. 1974, PASP, 86, 281

Boyd, M. R., Henry, T. J., Jao, W.-C., Subasavage, J. P., \& Hambly, N. C. 2011, AJ, 142, 92

Brandt, T. D., \& Huang, C. X. 2015, ApJ, 807, 58

Burgasser, A. J., Logsdon, S. E., Gagné, J., et al. 2015, ApJS, 220, 18

Chabrier, G., \& Baraffe, I. 2000, ARA\&A, 38, 337

Chabrier, G., Baraffe, I., \& Plez, B. 1996, ApJ, 459, L91

Chapin, E. L., Berry, D. S., Gibb, A. G., et al. 2013, MNRAS, 430, 2545

Chen, C. C., Cowie, L. L., Barger, A. J., et al. 2013, ApJ, 776, 13

Crifo, F., Phan-Bao, N., Delfosse, X., et al. 2005, A\&A, 441, 653

Cruz, K. L., \& Reid, I. N. 2002, AJ, 123, 2828

Deacon, N. R., \& Hambly, N. C. 2007, A\&A, 468, 163

Dempsey, J. T., Friberg, P., Jenness, T., et al. 2013, MNRAS, 430, 2534

Dent, W. R. F., Walker, H. J., Holland, W. S., \& Greaves, J. S. 2000, MNRAS, 314, 702

Deshpande, R., Martín, E. L., Montgomery, M. M., et al. 2012, AJ, 144, 99

Faherty, J. K., Burgasser, A. J., Walter, F. M., et al. 2012, ApJ, 752

Favata, F., Micela, G., \& Sciortino, S. 1997, A\&A, 322, 131

Filippazzo, J. C., Rice, E. L., Faherty, J., et al. 2015, ApJ, 810, 158

Finch, C. T., Henry, T. J., Subasavage, J. P., Jao, W.-C., \& Hambly, N. C. 2007 AJ, 133, 2898

Gagné, J., Lafrenière, D., Doyon, R., Malo, L., \& Artigau, E. 2014, ApJ, 783, 121

Gagné, J., Lafrenière, D., Doyon, R., Malo, L., \& Artigau, E. 2015, ApJ, 798, 73 Gálvez-Ortiz, M. C., Clarke, J. R. A., Pinfield, D. J., et al. 2010, MNRAS, 409, 552

Gliese, W., \& Jahreiß, H. 1991, VizieR Online Data Catalog: V/70A

Hambly, N. C., Henry, T. J., Subasavage, J. P., Brown, M. A., \& Jao, W.-C. 2004, AJ, 128, 437

Henry, T. J., Subasavage, J. P., Brown, M. A., et al. 2004, AJ, 128, 2460

Holland, W. S., Bintley, D., Chapin, E. L., et al. 2013, MNRAS, 430, 2513

Jenness, T., Berry, D., Chapin, E., et al. 2011, ASP Conf. Proc., 442, 281

Lépine, S. 2008, AJ, 135, 2177

Lestrade, J.-F., Wyatt, M. C., Bertoldi, F., Dent, W. R. F., \& Menten, K. M. 2006, A\&A, 460, 733

Luyten, W. J. 1979, Catalogue of stars with proper motions exceeding 0'.5 annually (LHS) (Minneapolis: University of Minnesota)

Lyo, A-R., Lawson, W. A., \& Bessell, M. S. 2004, MNRAS, 355, 363

Magazzù A., Martín, E. L., \& Rebolo, R. 1993, ApJ, 404, L17

Malo, L., Doyon, R., Lafrenière, D., et al. 2013, ApJ, 762, 88

Martín, E. L., Rebolo, R., \& Magazzù A. 1994, ApJ, 436, 262

Martín, E. L., Rebolo, R., \& Zapatero Osorio, M. R. 1996, ApJ, 469, 706

Martín, E. L., Basri, G., \& Zapatero Osorio, M. R. 1999a, AJ, 118, 1005

Martín, E. L., Delfosse, X., Basri, G., et al. 1999b, AJ, 118, 2466

Martín, E. L., Phan-Bao, N., Bessell, M., et al. 2010, A\&A, 517, A53

McLean, M., Berger, E., \& Reiners, A. 2012, ApJ, 746, 23

Messina, S., Lanzafame, A. C., Feiden, G. A., et al. 2016, A\&A, 596, A29

Montagnier, G., Ségransan, D., Beuzit, J.-L., et al. 2006, A\&A, 460, L19

Murphy, S. J., \& Lawson, W. A. 2015, MNRAS, 447, 1267

Pavlenko, Y. V., Rebolo, R., Martín E. L., \& García López, R. J. 1995, A\&A, 303, 807 
N. Phan-Bao et al.: Lithium in nearby, young late-M dwarfs

Phan-Bao N., \& Bessell M. S. 2006, A\&A, 446, 515

Phan-Bao N. Guifert, J., Crifo, F., et al. 2001, A\&A, 380, 590

Phan-Bao N., Crifo, F., Delfosse, X., et al. 2003, A\&A, 401, 959

Phan-Bao, N., Martín, E. L., Reylé, C., Forveille, T., \& Lim, J. 2005, A\&A, 439, L19

Phan-Bao, N., Bessell, M. S., Martín, E. L., et al. 2006a, MNRAS, 366, L40

Phan-Bao, N., Forveille, T., Martín, E. L., \& Delfosse, X. 2006b, ApJ, 645, L153

Phan-Bao, N., Bessell, M. S., Martín, E. L., et al. 2008, MNRAS, 383, 831

Phan-Bao, N., Lim, J., Donati, J.-F., Johns-Krull, C. M., \& Martín, E. L. 2009, ApJ, 704, 1721

Pokorny, R. S., Jones, H. R. A., \& Hambly, N. C. 2003, A\&A, 397, 575

Rajpurohit, A. S., Reylé, C., Allard, F., et al. 2013, A\&A, 556, A15

Rebolo R., Martín, E. L., \& Magazzù, A. 1992, ApJ, 389, L83

Rebolo R., Zapatero Osorio, M. R., \& Martín, E. L. 1995, Nature, 377, 129

Rebolo R., Martín, E. L., Basri, G., Marcy, G. W., \& Zapatero Osorio, M. R. 1996, ApJ, 469, L53

Reid, I. N., Kirkpatrick, J. D., Liebert, J., et al. 2002, AJ, 124, 519

Reiners, A., \& Basri, G. 2009, ApJ, 705, 1416

Reiners, A., Seifahrt, A., \& Dreizler, S. 2010, A\&A, 513, L9
Schmidt, S. J., Cruz, K. L., Bongiorno, B. J., Liebert, J., \& Reid, I. N. 2007, AJ, 133,2258

Schlieder, J. E., Lépine, S., Rice, E., et al. 2012, AJ, 143, 114

Shirley, Y. L., Huard, T. L., Pontoppidan, K. M., et al. 2011, ApJ, 728, 143

Shkolnik, E. L., Liu, M. C., \& Reid, I. N. 2009, ApJ, 699, 649

Shkolnik, E. L., Anglada-Escudé, G., Liu, M. C., et al. 2012, ApJ, 758, 56

Shortridge, K., Meyerdierks, H., Currie, M., et al. 2004, Starlink User Note 86

Song, I., Bessell, M. S., \& Zuckerman, B. 2002, ApJ, 581, L43

Subasavage, J. P., Henry, T. J., Hambly, N. C., Brown, M. A., \& Jao, W.-C. 2005, AJ, 129, 413

Thackrah, A., Jones, H., \& Hawkins, M. 1997, MNRAS, 284, 507

Tinney, C. G. 1998, MNRAS, 296, L42

Torres, C. A. O., Quast, G. R., Melo, C. H. F., \& Sterzik, M. F. 2008, in Young Nearby Loose Associations, Vol. I ed. B. Reipurth (The Southern Sky ASP Monograph Publications; San Francisco, CA: ASP), 757

West, A. A., Hawley, S. L., Bochanski, J. J., et al. 2008, AJ, 135, 785

West, A. A., Morgan, D. P., Bochanski, J. J., et al. 2011, AJ, 141, 97

Wroblewski, H., \& Torres, C. 1991, A\&AS, 91, 129

Wyatt, M. C. 2008, ARA\&A, 46, 339 